\title{
Massive expansions of Dscam splicing diversity via staggered homologous recombination during arthropod evolution
}

\author{
CHRISTOPHER LEE, ${ }^{1}$ NAMSHIN KIM, ${ }^{1,3,4}$ MEENAKSHI ROY, ${ }^{1,3}$ and BRENTON R. GRAVELEY ${ }^{2}$ \\ ${ }^{1}$ Department of Chemistry and Biochemistry, Center for Computational Biology, Institute for Genomics and Proteomics, Molecular Biology \\ Institute, University of California at Los Angeles, Los Angeles, California 90095-1570, USA \\ ${ }^{2}$ Department of Genetics and Developmental Biology, University of Connecticut Stem Cell Institute, University of Connecticut Health Center, \\ Farmington, Connecticut 06030-3301, USA
}

\begin{abstract}
The arthropod Down syndrome cell adhesion molecule (Dscam) gene can generate tens of thousands of protein isoforms via combinatorial splicing of numerous alternative exons encoding immunoglobulin variable domains organized into three clusters referred to as the exon 4, 6, and 9 clusters. Dscam protein diversity is important for nervous system development and immune functions. We have performed extensive phylogenetic analyses of Dscam from 20 arthropods (each containing between 46 and 96 alternative exons) to reconstruct the detailed history of exon duplication and loss events that built this remarkable system over 450 million years of evolution. Whereas the structure of the exon 4 cluster is ancient, the exon 6 and 9 clusters have undergone massive, independent expansions in each insect lineage. An analysis of nearly 2000 duplicated exons enabled detailed reconstruction of the timing, location, and boundaries of these duplication events. These data clearly show that new Dscam exons have arisen continuously throughout arthropod evolution and that this process is still occurring in the exon 6 and 9 clusters. Recently duplicated regions display boundaries corresponding to a single exon and the adjacent intron. The boundaries, homology, location, clustering, and relative frequencies of these duplication events strongly suggest that staggered homologous recombination is the major mechanism by which new Dscam exons evolve. These data provide a remarkably detailed picture of how complex gene structure evolves and reveal the molecular mechanism behind this process.
\end{abstract}

Keywords: alternative splicing; Dscam; evolution

\section{INTRODUCTION}

Alternative splicing is a powerful means of increasing protein diversity and regulating gene expression (Black 2000; Graveley 2001). Up to 95\% of human genes encode multiple mRNA isoforms (Mironov et al. 1999; Brett et al. 2000; Kan et al. 2001; Modrek et al. 2001; Pan et al. 2008;

\footnotetext{
${ }^{3}$ These authors contributed equally to this work.

${ }^{4}$ Present address: Korea Research Institute of Bioscience and Biotechnology (KRIBB), Yuseong-gu, Daejeon 305-806, Korea.

Reprint requests to: Christopher Lee, Department of Chemistry and Biochemistry, Center for Computational Biology, Institute for Genomics and Proteomics, Molecular Biology Institute, University of California at Los Angeles, Los Angeles, CA 90095-1570, USA; e-mail: leec@chem.ucla. edu; fax: (310) 206-4038; or Brenton R. Graveley, Department of Genetics and Developmental Biology, University of Connecticut Stem Cell Institute, University of Connecticut Health Center, 263 Farmington Avenue, Farmington, CT 06030-3301, USA; e-mail: graveley@neuron.uchc.edu; fax: (860) 679-8345.

Article published online ahead of print. Article and publication date are at http://www.rnajournal.org/cgi/doi/10.1261/rna.1812710.
}

Wang et al. 2008). Despite the abundance of alternative splicing in metazoans, relatively little is known about how alternatively spliced exons evolve. Known mechanisms of exon creation include the acquisition of mutations or sequences within introns that create new splice sites (LevMaor et al. 2003; Sorek et al. 2004; Alekseyenko et al. 2007) and the duplication of exons within a gene (Kondrashov and Koonin 2001; Letunic et al. 2002). While some exon duplication events have been identified, the number of these is so low that it has not been possible to determine the detailed mechanisms that led to their duplication (Letunic et al. 2002).

The arthropod Down syndrome cell adhesion molecule (Dscam) gene is remarkable in its capacity to encode tens of thousands of distinct isoforms through the process of alternative splicing (Schmucker et al. 2000; Zipursky et al. 2006). In Drosophila melanogaster, Dscam contains 95 alternatively spliced exons organized into four clusters referred to as the exon $4,6,9$, and 17 clusters that contain 
$12,48,33$, and two exons, respectively. The variable exons within each cluster are spliced in a mutually exclusive manner and they are independent of one another (Neves et al. 2004). As a result, Drosophila melanogaster potentially synthesizes 38,016 distinct Dscam mRNA isoforms, each of which encodes a distinct protein.

While the mechanism of mutually exclusive splicing has been studied in both the exon 4 (Kreahling and Graveley 2005) and exon 6 clusters (Graveley 2005; Anastassiou et al. 2006; Olson et al. 2007), the mechanism is only well understood for the exon 6 cluster. Mutually exclusive splicing within the exon 6 cluster involves the formation of competing RNA secondary structures between selector sequences located upstream of each variable exon and a single docking site located between exon 5 and the first exon 6 variant (Graveley 2005; Anastassiou et al. 2006). Importantly, as only one selector sequence can interact with the docking site at a time, the docking site-selector sequence interactions likely play a central role in the mutually exclusive splicing mechanism. A second component of this system involves the RNA binding protein hrp36, which binds to the exons throughout the exon 6 cluster and represses their inclusion (Olson et al. 2007). Based on these two observations, it has been proposed that the docking site-selector sequence interaction plays a positive role in the inclusion of a specific exon, while hrp36 functions globally to repress the inclusion of the other exons within the exon 6 cluster (Olson et al. 2007).

In neurons, the Dscam protein functions as an axon guidance receptor that determines the specificity of neural wiring through homophilic binding (i.e., a specific Dscam isoform binds to itself as a homodimer but does not bind to other isoforms) (Wang et al. 2002; Hummel et al. 2003; Zhan et al. 2004; Chen et al. 2006; Hattori et al. 2007; Hughes et al. 2007; Matthews et al. 2007; Soba et al. 2007). It is also expressed in hemocytes, where it appears that the encoded proteins act as immune receptors and interact with pathogens much like vertebrate antibodies (Watson et al. 2005; Dong et al. 2006).

The Dscam gene has several extraordinary features. First, the number of isoforms that Dscam can potentially generate by alternative splicing are at least an order of magnitude larger than any other known gene in any organism (Park and Graveley 2007). Second, the extensive alternative splicing of Dscam initially identified in D. melanogaster (Schmucker et al. 2000) has only been found to occur in insects (Graveley et al. 2004; Crayton et al. 2006) and crustaceans (Brites et al. 2008) and therefore appears to be restricted to the arthropod lineage. Third, the different number of variable exons present in each species suggests that Dscam is undergoing rapid evolution, especially in the exon 6 and 9 clusters (Graveley et al. 2004; Crayton et al. 2006). Given the important role of Dscam in both the nervous and immune systems, we have carried out an extensive evolutionary analysis of Dscam in 20 arthropod species. These studies have allowed us to characterize the patterns of evolution in each variable exon cluster and to determine the mechanism by which new Dscam exons are created and lost during evolution.

\section{RESULTS AND DISCUSSION}

\section{Organization of Dscam in 20 arthropod genomes}

To study the evolutionary history of Dscam in detail we compared the organization of the gene from 20 arthropod species. The species we examined consisted of one species of the order Crustacea (Daphnia pulex) and 19 species representing four major clades of the order Insecta. The insects studied include 12 Drosophila species (D. melanogaster, D. simulans, D. sechelia, D. yakuba, D. erecta, D. ananassae, D. pseudoobscura, D. persimilis, D. willistoni, D. mojavensis, D. virilis, and D. grimshawii), three mosquito species (Anopheles gambiae, Aedes aegypti, and Culex pipiens), two Hymenopteran species (Apis mellifera and Nasonia vitripennis), one Coleopteran (Tribolium castaneum), and one Lepidopteran (Bombyx mori). Together, these organisms allow us to examine Dscam evolution over $\sim 450-500$ million years, with both short-term resolution (comparing the organization of the gene within the 12 Drosophila or three mosquito species) and long-term resolution (comparing the organization of the gene between the four insect clades and Daphnia) (Fig. 1).

The overall organization of Dscam is very similar in the 12 Drosophila species (Fig. 1). The constitutive exons are highly conserved and have orthologs in each Drosophila species (Supplemental Fig. S1). Similarly, all Drosophila species contain 12 exon 4 variants and two exon 17 variants that are orthologous in each species. In contrast, the number of variable exons in the exon 6 and 9 clusters differs from species to species. The exon 6 cluster is the most variable and ranges from 41 exons in D. yakuba to 52 exons in D. virilis while the exon 9 cluster contains between 29 and 33 exons.

The organization of Dscam varies considerably between more distantly related species, though all species contain two exon 17 variants, except for D. pulex, which contains only one (Fig. 1; Brites et al. 2008). In contrast, the number of variable exons within the exon 4,6 , and 9 clusters varies significantly. For example, the number of variable exons ranges from seven to 15 in the exon 4 cluster, from 22 to 52 in the exon 6 cluster, and from 17 to 42 in the exon 9 cluster. Accordingly, the number of distinct protein ectodomains encoded by Dscam also varies considerably, from 2618 in D. pulex to 19,968 in D. virilis. Nonetheless, each species has the capacity to express an extraordinarily diverse repertoire of Dscam isoforms, suggesting that the Dscam combinatorial diversity is functionally important and ancient, predating the arthropod ancestor. However, it is difficult to reconstruct the evolutionary history of this structure beyond the insect ancestor; Crayton et al. (2006) 

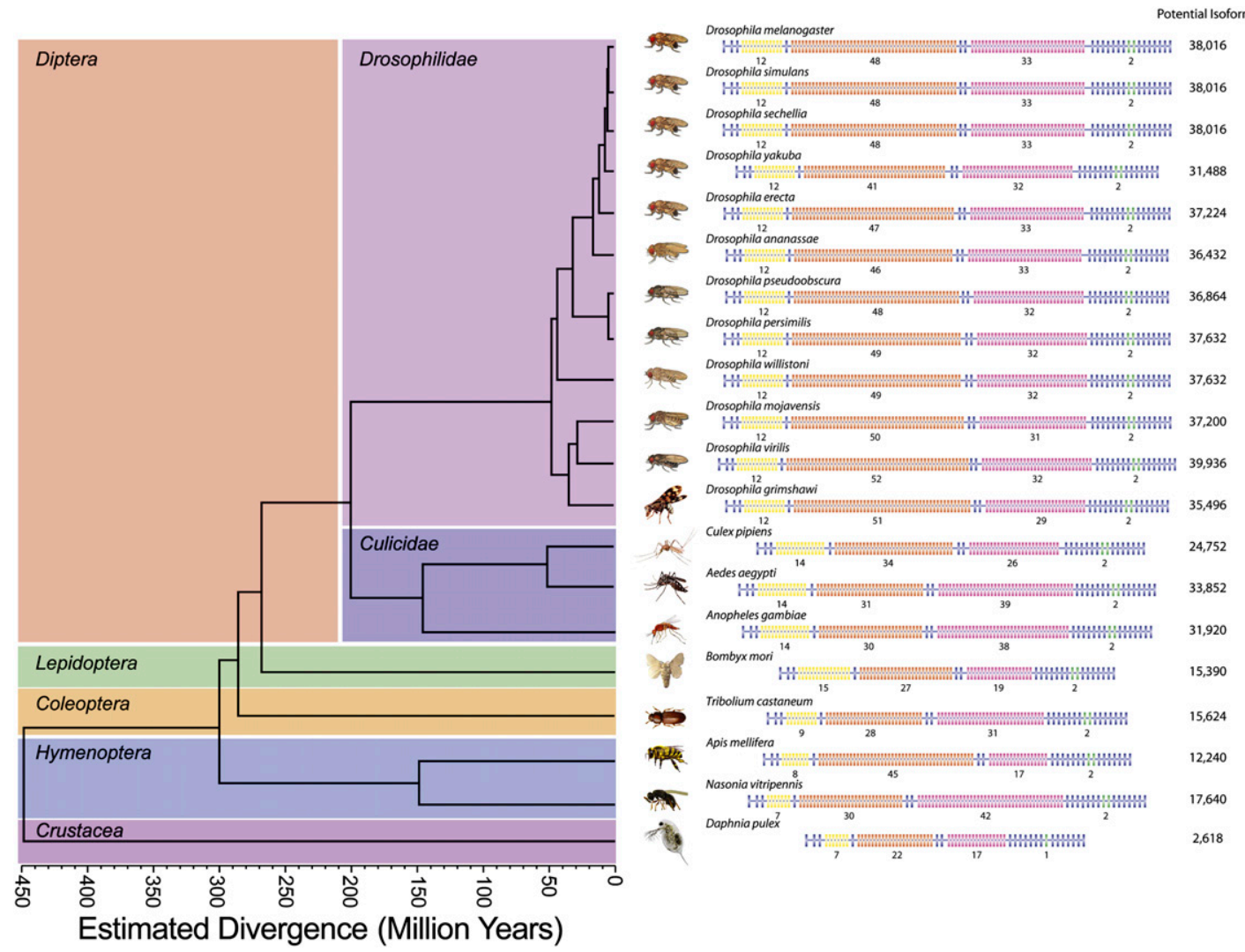

FIGURE 1. Evolution of Dscam in 20 Arthropod genomes. Phylogenetic tree and Dscam gene structures of 12 Drosophila, three mosquito, silkworm (Bombyx mori), beetle (Tribolium castenneum), honeybee (Apis mellifera), wasp (Nasonia vitripennis), and waterflea (Daphnia pulex) species. The number of exons in each alternatively spliced cluster is annotated below the cluster. The total number of potential isoforms is indicated on the right. A phylogenetic tree depicting the relationships between each species is shown on the left.

found no convincing shared phylogeny between any specific insect exon 4,6 , or 9 versus the corresponding exon sequences from insect Dscam homologs 1, 2, and 3, and from vertebrate $d s c a m$ and $d s c a m L$.

A nucleotide alignment of the Dscam genes from these 20 species, using $D$. melanogaster as a reference, reveals that the constitutive exons are highly conserved across all species (Supplemental Fig. S1). However, the sequence of the variable exons within the exon 4, 6, and 9 clusters has diverged considerably and is most apparent in the exon 6 and 9 clusters. For example, despite the existence of comparable numbers of exons in the exon 6 clusters of D. melanogaster and $A$. mellifera, the alternative exons from these species share very little nucleotide sequence similarity.

\section{Global analysis of variable exon relationships}

As an overview of the evolutionary relationships among the variable exons, we generated heatmaps for each cluster to show the relative similarity of each variable exon to all other variable exons both within the same species and between species (Fig. 2). For these analyses we selected one species to represent each of the major clades being
studied-D. melanogaster, A. gambiae, B. mori, T. castaneum, A. mellifera, and D. pulex. For each cluster, we calculated the pairwise percentage of identity of each variable exon to all other variable exons. We then converted the percentage identities into $Z$-scores and projected these as heatmaps, where yellow indicates the most similar pairs of exons and blue indicates the least similar pairs of exons. To simplify visualization, the exons are depicted in the heat maps in the same linear order in which they reside in the gene.

Three striking features of the exon 4 cluster emerge from this analysis (Fig. 2A). First, with the exception of B. mori and $D$. pulex, most exons within one species are orthologous to exons in the other species. Second, these orthologous exons are typically organized in approximately the same linear order in the gene, as revealed by the yellow diagonals in the regions of the heatmap that compare two species (e.g., D. melanogaster and T. castaneum). Third, we noted that in some cases the most highly similar pairs of exons are found within a species (especially B. mori), not between species-in other words, such paralog duplications have occurred after that species diverged from other species within the tree. Furthermore, paralogous exons 
tend to be located adjacent to one another in the gene, as shown by the bright yellow boxes just off the diagonal in the heatmap. For instance, in B. mori, the last six exons are significantly more similar to one another than to any of the 50 exons in the other five species. This suggests that these six B. mori exons are the products of a series of duplications that occurred specifically in the Lepidopteran lineage.

Analysis of the heatmaps of the exon 6 (Fig. 2B) and exon 9 (Fig. 2C) clusters reveal a dramatically different pattern. There is little evidence supporting the existence of conserved orthologous pairs of exons between species in the exon 6 and exon 9 clusters. Instead, when strong similarities exist between exons in different species (e.g., exons 6.36-6.46 in D. melanogaster and exons 6.7-6.26 in A. gambiae), these involve large blocks of highly similar exons within each species, rather than pairs of exons, suggesting that large expansions of an ancestral exon occurred independently in each lineage. These results are in general agreement with published trees of the exon 6 and 9 clusters for D. melanogaster, A. gambiae, and A. mellifera (Graveley et al. 2004; Crayton et al. 2006). Whereas the exon 6 cluster contains a number of such "common blocks" that span multiple insect clades, within the exon 9 cluster, there are very few cases of exons from different species that are related to one another. Rather, the heatmap of the exon 9 cluster consists almost exclusively of large blocks of duplications that are specific to each clade (Fig. 2C). For example, a massive block of 24 exons expanded specifically within the A. gambiae lineage. Similarly, in D. melanogaster, there is a block of 13 exons that are highly similar to one another. As with the exon 4 cluster, the most similar exons within each species tend to be adjacent to one another.

\section{Minimal reconstruction of ancestral Dscam exon structure}

We next conducted a detailed analysis of the evolutionary history of each variable exon cluster. To do this we constructed multiple sequence alignments of all of the exons within each cluster from the 20 species and generated a phylogenetic tree for each cluster by neighbor joining (Saitou and Nei 1987). Based on these phylogenies, we inferred the evolutionary history of each cluster and the minimal exon structure of important ancestors in the tree (e.g., the ancestor of all insects) using standard phylogenetic principles: orthologs, paralogs, and monophyletic groups (Supplemental Fig. S2; Maddison 1997). Briefly, if two exons in different species are more closely related to each other than to other exons in the same species (according to the phylogenetic analysis), we infer that they descended from an ancestral exon present in the most recent common ancestor (MRCA). Exons with this property are referred to as orthologous pairs of exons. Alternatively, if two exons in a single species are more closely related
A.

\section{Exon 4}

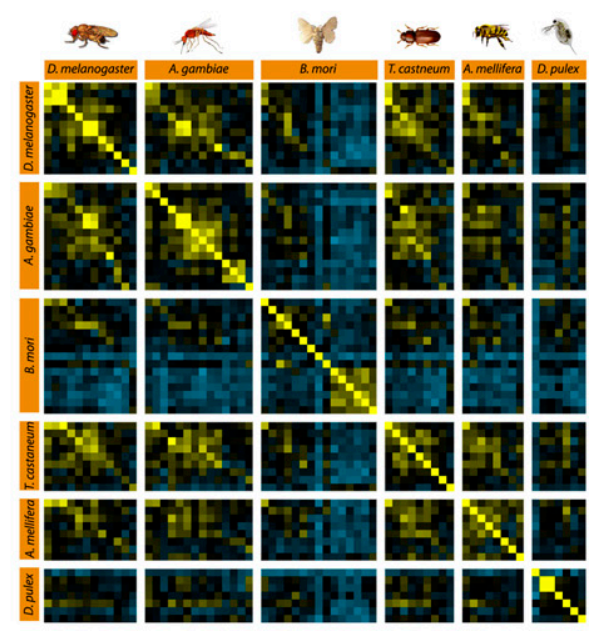

B.

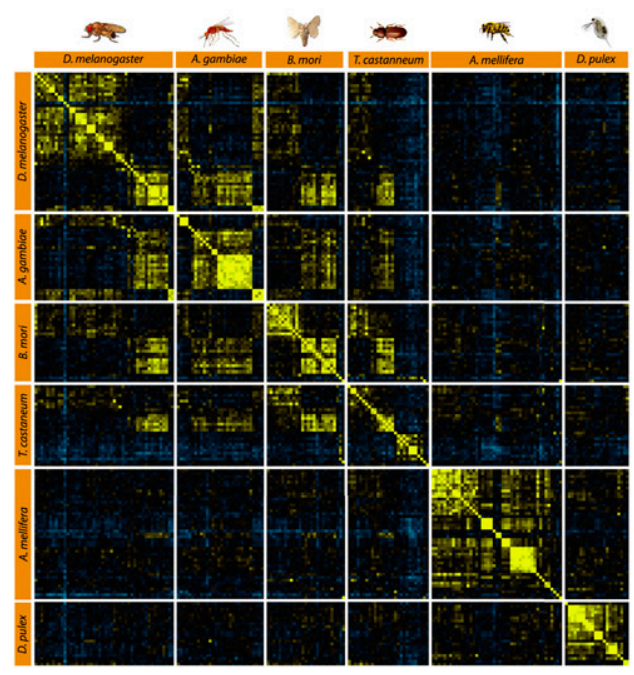

C.

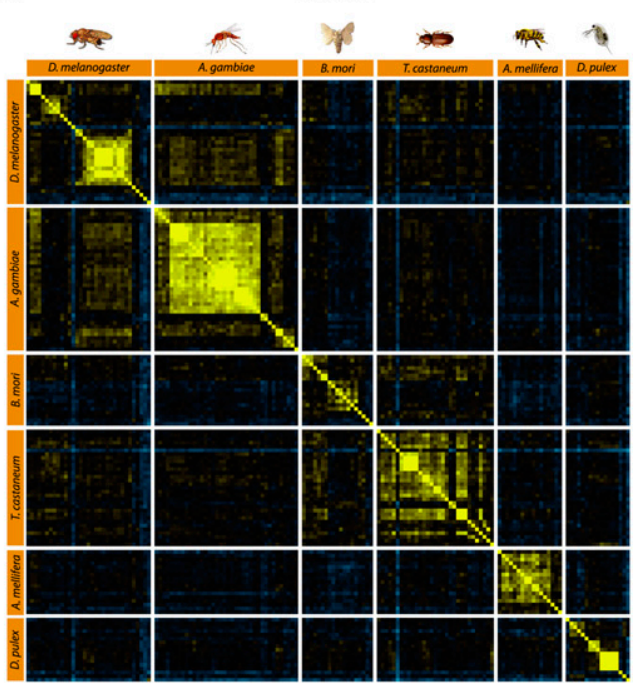

FIGURE 2. (Legend on next page) 
to each other than to any exons in other species, we infer that they resulted from a duplication event that occurred during the evolution of that species after divergence from the MRCA. Exons having this relationship are referred to as paralogous exons. Indeed, any phylogenetic branch that connects two exons from the same species is direct evidence of a recent duplication event. Finally, a monophyletic group is a subset of exons in a tree that constitutes all descendants of their MRCA in the tree (Supplemental Fig. S2). Such groups can be used to identify crossconnections between clades that indicate the specific set of exons that were present in their MRCA (for details, see Supplemental Fig. S2; Materials and Methods). To analyze the statistical significance of the phylogeny results, we ran 1000 phylogeny analyses using standard bootstrap tree sampling and identified groups of exons that were reported as monophyletic groups with high probability in the bootstrap sampling (Table 1-3; for details, see Materials and Methods).

For example, using Diptera as the ingroup, all Drosophila exons $4.1-4.3$ and all mosquito exons $4.1-4.2$ formed a monophyletic group in $60 \%$ of bootstrap runs, and in nearly all of those cases three outgroup exons- $-T$. castaneum exon 4.1, A. mellifera 4.1, and N. vitripennis 4.1-clustered with them in the bootstrap trees (Table 1, exon A). The fact that these outgroup exons preferentially cluster with this group, and not with some higher grouping of Diptera exons, implies that both the ingroup and outgroup exons descended from one exon present in the Insecta ancestor.

This analysis indicates that the structure of the exon 4 cluster is ancient and is largely unchanged since the Insecta ancestor (Fig. 3; Supplemental Fig. S3). Our monophyletic analysis indicates that at least nine exons were present in the Insecta ancestor, nearly all of which are preserved in each modern insect species (Supplemental Fig. S3; Table 1). Indeed, the $T$. castaneum exon 4 structure appears to be essentially identical to the Insecta ancestral structure. These conclusions are further supported by the fact that the linear

FIGURE 2. Exon similarity within the exons 4, 6, and 9 clusters in representative species from each of the major arthropod clades. $(A)$ The percentage of identity for all possible pairs of variable exons from the exon 4 clusters of $D$. melanogaster, A. gambiae, A. mellifera, B. mori, T. castaneum, and D. pulex were determined using ClustalW. $Z$-scores of the percentage identity for each pair of exons were then calculated using the average and standard deviation of all pairwise values. The $Z$-score values were then represented as heat maps in which the order of the exons in the heatmap corresponds to the linear order of each exon in the genome. Yellow indicates a high $Z$-score, and therefore a high percent identity, while blue indicates a low $Z$-score and, thus, little sequence similarity. (B) Heatmaps of the $Z$-scores of the percent identities of all exons in the exon 6 clusters from D. melanogaster, A. gambiae, A. mellifera, B. mori, T. castaneum, and $D$. pulex calculated as described above. (C) Heatmaps of the Z-scores of the percentage of identities of all exons in the exon 9 clusters from D. melanogaster, A. gambiae, A. mellifera, B. mori, T. castaneum, and D. pulex calculated as described above. order of the orthologous exons is identical in all insect species (Supplemental Fig. S3). For example, the first exon in T. castaneum (exon 4.1) is orthologous to the first exons in D. melanogaster, while the last exon in T. castaneum (exon 4.9) is orthologous to the last exon in D. melanogaster (exon 4.12) (Table 1). This is true for all sets of orthologous exons in all of the species analyzed. It is possible that the apparent absence of clear orthologs for exons B, C, E, G, H, and I in B. mori is simply a problem of insufficient confidence, since exons are present at each of those positions in B. mori but could not be assigned as orthologous (Table 1; Supplemental Fig. S3).

Our analyses of the exon 6 and 9 clusters permit only a more limited ancestral reconstruction. For example, in the exon 9 cluster, only two groups bridged across InsectaCrustacea clades according to our monophyletic analysis (Table 3). This could mean that all other exons in the exon 9 cluster are derived from recent duplications (i.e., the Insecta ancestor had only two exons in the exon 9 cluster). This represents a "minimal reconstruction" of the ancestral structure limited to the cases where the evidence exceeded our detection thresholds. Alternatively, it is possible that additional exons were present in the ancestral exon 9 cluster but have simply diverged too much to reconstruct their phylogenetic relations with adequate confidence. Regardless, there is clear evidence of major, independent expansions in each clade. For example, the evolution of the exon 9 cluster involved serial duplications of a single ancestral exon to produce 25 exons in the modern Culicidae genomes (Fig. 3 , represented by the large green pie slice; for details, see Supplemental Fig. S5E). By contrast, the two exons inferred to be present in the Diptera ancestor (Fig. 3, represented by the blue pie slice in the Diptera ancestor) have given rise to only six exons in modern Culicidae genomes. In the Drosophilidae, a different ancestral exon dominated, giving rise to 17 modern exons, while other ancestral exons are represented by two, four, and seven modern exons, respectively (Fig. 3; Supplemental Fig. S5D). Similarly, in N. vitripennis, one ancestral exon duplicated to produce 16 exons in the exon 9 cluster (Fig. 3, large green sector). In the exon 6 cluster, we detected evidence of six ancestral exons that span the major insect clades (i.e., they were present in the Insecta ancestor) (Table 2; Supplemental Fig. S4). As in the exon 9 cluster, major expansions appear to have occurred in the exon 6 cluster independently in each insect clade (Supplemental Fig. S4). Overall, these data indicate that the exon 6 and 9 clusters expanded significantly more than the exon 4 cluster during insect evolution.

Our data suggest that the Insecta ancestor contained at least eight exon 4 variants, six exon 6 variants, two exon 9 variants, and two exon 17 variants and therefore could potentially encode at least 192 isoforms. However, given the rapid evolution of the variable exon clusters, and the fact that this reconstructed minimal ancestral gene structure only includes high confidence exons, it is likely that the 
TABLE 1. Cross-clade phylogenetic connections: The exon 4 cluster

\begin{tabular}{|c|c|c|}
\hline Monophyletic group (ingroup) & \%Monophyletic & Outgroup \\
\hline $\begin{array}{l}\text { Exon A: ANA4.1-4.3, ERE4.1-4.3, GRM4.1-4.3, MEL4.1-4.3, } \\
\text { MOJ4.1-4.3, PER4.1-4.3, PSE4.1-4.3, SEC4.1-4.3, } \\
\text { SIM4.1-4.3, VIR4.1-4.3, WIL4.1-4.3, YAK4.1-4.3, } \\
\text { AEG4.1-4.2, GAM4.1-4.2, PIP4.1-4.2 }\end{array}$ & $59.6 \%$ & CAS4.1 (59.5\%), MLF4.1 (57.8\%), VIT4.1 (57.8\%) \\
\hline Exon A: MLF4.1, VIT4.1 & $100.0 \%$ & $\begin{array}{l}\text { CAS4.1 (95.7\%), dros4.3 (95.5\%), dros4.1 (95.3\%), dros4.2 } \\
(95.2 \%), \text { GAM4.2 (89.7\%), PIP4.2 (88.9\%), AEG4.2 } \\
(88.9 \%), \text { MOR4.1 (75.4\%), PIP4.1 (68.7\%), AEG4.1 } \\
(68.7 \%), \text { GAM4.1 }(68.7 \%)\end{array}$ \\
\hline $\begin{array}{l}\text { Exon B: ANA4.4, ERE4.4, GRM4.4, MEL4.4, MOJ4.4, } \\
\text { PER4.4, PSE4.4, SEC4.4, SIM4.4, VIR4.4, WIL4.4, YAK4.4, } \\
\text { AEG4.3, PIP4.3, GAM4.3 }\end{array}$ & $81.8 \%$ & CAS4.2 $(73.6 \%)$ \\
\hline Exon B: MLF4.2, VIT4.2 & $100.0 \%$ & $\begin{array}{l}\text { YAK4.4 (55.6\%), VIR4.4 (55.6\%), SIM4.4 (55.6\%), SEC4.4 } \\
\text { (55.6\%), PSE4.4 (55.6\%), PER4.4 (55.6\%), MOJ4.4 } \\
(55.6 \%), \text { MEL4.4 (55.6\%), GRM4.4 (55.6\%), ERE4.4 } \\
\text { (55.6\%), ANA4.4 (55.6\%), WIL4.4 (55.5\%), AEG4.3 } \\
\text { (55.4\%), PIP4.3 (55.4\%), GAM4.3 (55.0\%), PUL4.5 } \\
\text { (54.2\%), CAS4.2 }(51.8 \%)\end{array}$ \\
\hline $\begin{array}{l}\text { Exon C: ANA4.5, ERE4.5, GRM4.5, MEL4.5, MOJ4.5, } \\
\text { PER4.5, PSE4.5, SEC4.5, SIM4.5, VIR4.5, WIL4.5, YAK4.5, } \\
\text { AEG4.4, PIP4.4, GAM4.4 }\end{array}$ & 95.2 & CAS4.3 (92.5\%), MLF4.3 (90.9\%), VIT4.3 (90.6\%) \\
\hline $\begin{array}{l}\text { Exon D: ANA4.6-4.7, ERE4.6-4.7, GRM4.6-4.7, MEL4.6-4.7, } \\
\text { MOJ4.6-4.7, PER4.6-4.7, PSE4.6-4.7, SEC4.6-4.7, } \\
\text { SIM4.6-4.7, VIR4.6-4.7, WIL4.6-4.7, YAK4.6-4.7, } \\
\text { AEG4.5-4.6, GAM4.5-4.6, PIP4.5-4.6 }\end{array}$ & $94.1 \%$ & MOR4.4 (82.8\%), CAS4.4 (65.4\%) \\
\hline $\begin{array}{l}\text { Exon E: ANA4.8, ERE4.8, GRM4.8, MEL4.8, MOJ4.8, } \\
\text { PER4.8, PSE4.8, SEC4.8, SIM4.8, VIR4.8, WIL4.8, YAK4.8 }\end{array}$ & $100.0 \%$ & CAS4.5 (51.1\%) \\
\hline $\begin{array}{l}\text { Exon F: ANA4.9, ERE4.9, GRM4.9, MEL4.9, MOJ4.9, } \\
\text { PER4.9, PSE4.9, SEC4.9, SIM4.9, VIR4.9, WIL4.9, YAK4.9, } \\
\text { AEG4.10, PIP4.10, GAM4.10 }\end{array}$ & $93.9 \%$ & MOR4.6 (54.7\%), CAS4.6 (63.4\%) \\
\hline $\begin{array}{l}\text { Exon G: ANA4.10, ERE4.10, GRM4.10, MEL4.10, MOJ4.10, } \\
\text { PER4.10, PSE4.10, SEC4.10, SIM4.10, VIR4.10, WIL4.10, } \\
\text { YAK4.10, AEG4.11-4.13, GAM4.11-4.13, PIP4.11-4.13 }\end{array}$ & $70.7 \%$ & CAS4.7 (51.9\%) \\
\hline Exon G: MLF4.6 & & $\begin{array}{l}\text { ANA4.10 }(63.9 \%), \text { ERE4.10 }(63.9 \%), \text { GRM4.10 }(63.9 \%), \\
\text { MEL4.10 }(63.9 \%), \text { MOJ4.10 (63.9\%), PSE4.10 }(63.9 \%), \\
\text { SEC4.10 (63.9\%), SIM4.10 (63.9\%), VIR4.10 }(63.9 \%), \\
\text { WIL4.10 (63.9\%), YAK4.10 (63.9\%), CAS4.7 }(63.3 \%), \\
\text { GAM4.13 (62.0\%), PIP4.13 (61.9\%), AEG4.13 }(61.9 \%), \\
\text { PIP4.12 (58.3\%), GAM4.12 (58.3\%), AEG4.12 }(58.3 \%), \\
\text { PIP4.11 (54.5\%), GAM4.11 (54.5\%), AEG4.11 }(54.5 \%)\end{array}$ \\
\hline $\begin{array}{l}\text { Exon H: ANA4.11, ERE4.11, GRM4.11, MEL4.11, MOJ4.11, } \\
\text { PER4.11, PSE4.11, SEC4.11, SIM4.11, VIR4.11, WIL4.11, } \\
\text { YAK4.11, AEG4.14, PIP4.14, GAM4.14 }\end{array}$ & $95.3 \%$ & CAS4.8 (81.5\%), MLF4.7 (76.1\%), VIT4.6 (75.9\%) \\
\hline $\begin{array}{l}\text { Exon I: ANA4.12, ERE4.12, GRM4.12, MEL4.12, MOJ4.12, } \\
\text { PER4.12, PSE4.12, SEC4.12, SIM4.12, VIR4.12, WIL4.12, } \\
\text { YAK4.12 }\end{array}$ & $100.0 \%$ & CAS4.9 (93.6\%), MLF4.8 (91.6\%), VIT4.7 (91.6\%) \\
\hline
\end{tabular}

Insecta ancestor contained more exons, especially within the exon 6 and 9 clusters.

It is difficult to confidently reconstruct the organization of Dscam in the Pancrustacea ancestor. Nonetheless, we have identified several monophyletic group connections between Insecta and Daphnia: one in the exon 4 cluster and two in the exon 9 cluster (Tables 1, 3). These data suggest that at least two exon 9 variants were present in the Pancrustacea ancestor. However, since D. pulex contains large exon 4 and 6 clusters like insects, and since the mechanism of mutually exclusive splicing of the exon 6 cluster is conserved between $D$. pulex and insects, it seems likely that the Pancrustacea ancestor also contained at least two variable exons in both the exon 4 and 6 clusters.

\section{Ancestral duplication analysis over the insect phylogeny}

To analyze the evolutionary history leading to each exon in the modern species, we tracked the number of duplication events that occurred on the path to each exon since the Insecta ancestor (Fig. 4; Supplemental Fig. S5; for details, 
TABLE 2. Cross-clade phylogenetic connections: The exon 6 cluster

\begin{tabular}{|c|c|c|}
\hline Monophyletic group (ingroup) & \% Monophyletic & Outgroup \\
\hline $\begin{array}{l}\text { Exon A: ANA6.1, ERE6.1, GRM6.1, MEL6.1, MOJ6.1, PER6.1, } \\
\text { PSE6.1, SEC6.1, SIM6.1, VIR6.1, WIL6.1, YAK6.1 }\end{array}$ & $99.7 \%$ & 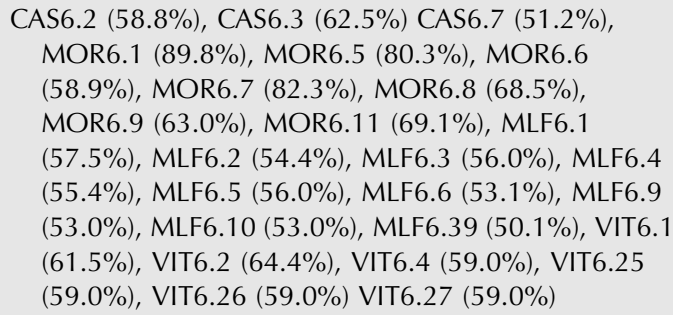 \\
\hline $\begin{array}{l}\text { Exon B: ANA6.31, ERE6.29, GRM6.35, MEL6.30, MOJ6.33, } \\
\text { PER6.30, PSE6.30, SEC6.29, SIM6.30, VIR6.35, WIL6.32, } \\
\text { YAK6.25 }\end{array}$ & $100.0 \%$ & CAS6.8 (74.5\%) \\
\hline Exon C: CAS6.14, CAS6.16 & $72.3 \%$ & MOR6.24 (60.3\%) \\
\hline Exon D: MLF6.43, VIT6.29 & $87.3 \%$ & CAS6.17 (72.4\%) \\
\hline Exon E: CAS6.18-6.26 & $57.4 \%$ & MOR6.26 (57.4\%) \\
\hline Exon E: MLF6.44, VIT6.30 & $57.1 \%$ & \\
\hline Exon E: MOR6.26 & $(100 \%)$ & $\begin{array}{l}\text { CAS6.18 }(94.6 \%), \text { CAS6.19 }(87.4 \%), \text { CAS6.20 }(94.6 \%), \\
\text { CAS6.21 }(87.4 \%), \text { CAS6.22 }(98.9 \%) \text {, CAS6.23 } \\
(99.0 \%), \text { CAS6.24 }(98.8 \%), \text { CAS6.25 }(89.1 \%), \\
\text { CAS6.26 }(94.5 \%), \text { MLF6.44 (70.6\%), VIT6.30 }(61.5 \%)\end{array}$ \\
\hline Exon F: MLF6.45 & $(100.0 \%)$ & CAS6.27 (76.8\%), MOR6.27 (87.4\%) \\
\hline $\begin{array}{l}\text { Exon q: ANA6.32-6.33, ERE6.30-6.31, ERE6.33, GRM6.36.-6.38, } \\
\text { GRM6.40, MEL6.31-6.32 MEL6.34, MOJ6.34-6.36, MOJ6.38, } \\
\text { PER6.31-6.32, PSE6.31-6.32, SEC6.30-6.31, SEC6.33, } \\
\text { SIM6.31-6.32, SIM6.34, VIR6.36.-6.38, VIR6.40, WIL6.33, } \\
\text { WIL6.35, WIL6.37, YAK6.26.-6.27, YAK6.29, AEG6.02, } \\
\text { GAM6.05, PIP6.05 }\end{array}$ & $69.5 \%$ & \\
\hline $\begin{array}{l}\text { Exon r: ANA6.34, ERE6.32, ERE6.34, GRM6.39, GRM6.41, } \\
\text { MEL6.33, MEL6.35, MOJ6.37, MOJ6.39, PER6.33-6.34, } \\
\text { PSE6.33-6.34, SEC6.32, SIM6.33, SIM6.35, VIR6.39, VIR6.41, } \\
\text { WIL6.36, WIL6.38, YAK6.28, YAK6.30, AEG6.03, GAM6.06, } \\
\text { PIP6.06 }\end{array}$ & $64.9 \%$ & \\
\hline $\begin{array}{l}\text { Exon s: ANA6.44-6.45, ERE6.45, GRM6.51-6.52, MEL6.47-6.48, } \\
\text { MOJ6.48-6.49, PER6.46.-6.47, PSE6.46.-6.47, SEC6.46.-6.47, } \\
\text { SIM6.47-6.48, VIR6.50-6.51, WIL6.48-6.49, YAK6.40 } \\
\text { AEG6.26-6.29, GAM6.27-6.30, PIP6.31-6.34 }\end{array}$ & $81.1 \%$ & \\
\hline Exon h: MLF6.8, MLF6.15, VIT6.3 & $85.6 \%$ & \\
\hline Exon i: MLF6.11, VIT6.05-6.06 & $91.0 \%$ & \\
\hline Exon j: MLF6.14, VIT6.07 & $86.4 \%$ & \\
\hline Exon k: MLF6.16, VIT6.08 & $74.4 \%$ & \\
\hline Exon I: MLF6.17, VIT6.09 & $81.5 \%$ & \\
\hline Exon m: MLF6.25-6.27, VIT6.10-6.12 & $55.9 \%$ & \\
\hline Exon n: MLF6.35, VIT6.14-6.15 & $82.0 \%$ & \\
\hline Exon o: MLF6.37, VIT6.17-6.18, VIT6.20-6.21 & $55.6 \%$ & \\
\hline Exon p: MLF6.42, VIT6.24, VIT6.28 & $97.6 \%$ & \\
\hline
\end{tabular}

see Materials and Methods). These results provide another example of the dramatic differences between the exon 4 cluster and the exon 6 and 9 clusters. The exon 4 cluster has a fairly stable and unchanging gene structure during insect evolution; with the exception of $B$. mori, there have been virtually no duplications on the lineages of most exon 4 variants. In contrast, the exon 6 and 9 clusters have undergone massive expansions via exon duplication over the same time period. In the exon 6 cluster, nearly all exons have experienced an average of two duplication events since the Insecta ancestor (Fig. 4). In fact, there are several exons that have experienced at least five duplications during that period (Supplemental Fig. S5). Moreover, within the exon 9 cluster, most exons have experienced between two and five duplications (Fig. 4), and several exons within A. gambiae have been duplicated as many as 10 times (Supplemental Fig. S5). Interestingly, while these expansions form contiguous blocks within each cluster (see also Fig. 2B,C), most duplications occur in the center of each cluster, with few or no duplications occurring at the edges of the clusters-the first and last exons in the exon 6 and 9 clusters generally have low duplication counts, typically zero or one (Fig. 4). 
TABLE 3. Cross-clade phylogenetic connections: The exon 9 cluster

\begin{tabular}{|c|c|c|}
\hline Monophyletic group (ingroup) & $\%$ Monophyletic & Outgroup \\
\hline $\begin{array}{l}\text { Exon a: ANA9.1-9.4, ERE9.1-9.4, } \\
\text { GRM9.1-9.4, MEL9.1-9.4, MOJ9.1-9.4, } \\
\text { PER9.1-9.4, PSE9.1-9.4, SEC9.1-9.4, } \\
\text { SIM9.1-9.4, VIR9.1-9.4, WIL9.1-9.4, } \\
\text { YAK9.1-9.4, GAM10.1-10.4, AEG10.1- } \\
\text { 10.6, PIP10.1-10.7 }\end{array}$ & $94.3 \%$ & \\
\hline $\begin{array}{l}\text { Exon b: ANA9.5-9.11, ERE9.5-9.11, } \\
\text { GRM9.5-9.11, MEL9.5-9.11, MOJ9.5- } \\
\text { 9.11, PER9.5-9.11, PSE9.5-9.11, } \\
\text { SEC9.5-9.11, SIM9.5-9.11, VIR9.5- } \\
\text { 9.11, WIL9.5-9.11, YAK9.5-9.11, } \\
\text { GAM10.29-10.30, PIP10.20 }\end{array}$ & $65.8 \%$ & \\
\hline $\begin{array}{l}\text { Exon B: ANA9.33, ERE9.33, GRM9.32, } \\
\text { MEL9.33, MOJ9.31, PER9.32, PSE9.32, } \\
\text { SEC9.33, SIM9.33, VIR9.32, WIL9.29, } \\
\text { YAK9.32 }\end{array}$ & $100.0 \%$ & PUL10.17 (58.1\%) \\
\hline Exon c: MLF10.1, VIT10.1 & $100.0 \%$ & \\
\hline Exon d: MLF10.2, VIT10.2 & $98.3 \%$ & \\
\hline Exon e: MLF10.3, VIT10.3 & $60.0 \%$ & \\
\hline Exon f: MLF10.7, VIT10.5 & $73.5 \%$ & \\
\hline Exon g: MLF10.8-10.9, VIT10.6 & $65.8 \%$ & \\
\hline $\begin{array}{l}\text { Exon h: MLF10.13, (VIT10.8 VIT10.14), } \\
\text { VIT10.23, VIT10.25, VIT10.27, } \\
\text { (VIT10.29 VIT10.31), VIT10.33, } \\
\text { VIT10.35 }\end{array}$ & $82.6 \%$ & \\
\hline Exon i: MLF10.15, VIT10.40 & $100.0 \%$ & \\
\hline Exon j: MLF10.16, VIT10.41 & $100.0 \%$ & \\
\hline Exon k: MLF10.17, VIT10.42 & $100.0 \%$ & \\
\hline Exon A: MOR10.19 & $100.0 \%$ & PUL10.15 (62.8\%) \\
\hline
\end{tabular}

the results of our detailed phylogenetic analysis from which we can draw several conclusions. First, the exon 6 and 9 clusters have undergone massive expansions via exon duplication over the last 300 million years. For example, there were eight duplications in the exon 6 cluster and 10 duplications in the exon 9 cluster during the divergence of Hymenoptera from the Insecta ancestor between 300 and 150 million years ago (mya). Similarly, over the ensuing 150 million years when $N$. vitripennis evolved from the Hymenoptera ancestor, there were 11 duplications in the exon 6 cluster and 29 duplications in the exon 9 cluster. Second, independent large expansions have occurred in all branches. For example, the divergence of the Diptera ancestor into the Drosophilidae and Culicidae ancestors involved 39 and 25 duplications, respectively, in the exon 6 cluster and involved 26 and 33 duplications, respectively, in the exon 9 cluster. Third, this expansion process is still ongoing as demonstrated by recent duplications in D. yakuba (one duplication within the exon 6 cluster during the last 5 million years), D. ananassae (one duplication in

This same pattern is observed for each duplication block. For example, D. melanogaster exons 9.14-9.30 form a contiguous block of highly similar exons plainly visible in the heatmap of the exon 9 cluster (Fig. 2C), with large numbers of duplications at the center of the block (a peak of seven duplications for exon 9.20) and zero duplications for exons at the boundaries of the block (exons 9.13, 9.31, 9.32, and 9.33) (Supplemental Fig. S6). Other duplication blocks follow the same basic pattern.

Interestingly, the boundaries of these duplication blocks often correlate with the spatial structure of the gene. For example, the low duplication density in D. melanogaster 6.30-6.39 corresponds to a region in the exon 6 cluster containing unusually long introns between exons 6.33, 6.34, 6.35 , and 6.36 (data not shown). This region also demarcates a very clear boundary between the two distinct homology blocks visible in the heatmap of the exon 6 cluster (Fig. 2B) indicative of two independently evolving segments of the exon 6 cluster. Taken together, these data suggest a duplication mechanism whose frequency of occurrence depends on the number of neighboring homology partners with which a given exon can pair.

We next sought to analyze the temporal distribution of duplication events over each distinct stage of evolution. Figure 5 (see also Supplemental Figs. S2-S4) summarizes both the exon 6 and 9 clusters during the last 13 million years), D. mojavensis (one duplication in the exon 6 cluster during the last 23 million years), and D. grimshawii (one duplication in the exon 6 cluster during the last 31 million years), to give a few examples. Fourth, there are substantial variations in the frequency of duplication per evolutionary time (e.g., 22 duplications in the exon 6 cluster on the $A$. mellifera branch but only five in the exon 9 cluster).

The large number of Dipteran species in this analysis allowed us to compare the frequency of duplication events in early versus more recent insect evolution (specifically, before and after the branching of Drosophilidae and 9 clusters have occurred at a rate of approximately one duplication per 10 million years (Fig. 6). The estimated frequency appears to be lower for very long branch times, as would be expected due to the increased probability that a duplicated exon will be lost before it can be observed (at the end of the branch). Note that by contrast the exon 4 cluster has been almost completely static over the same period-all Drosophilidae species contain 12 exon 4 variants and all three Culicidae species contain 14 exon 4 variants. Only two and four duplications were detected in the exon 4 cluster during the period 200-50 mya when the Drosophilidae and Culicidae, respectively, diverged from Culicidae). Overall, exon duplications in the exon 6 and 


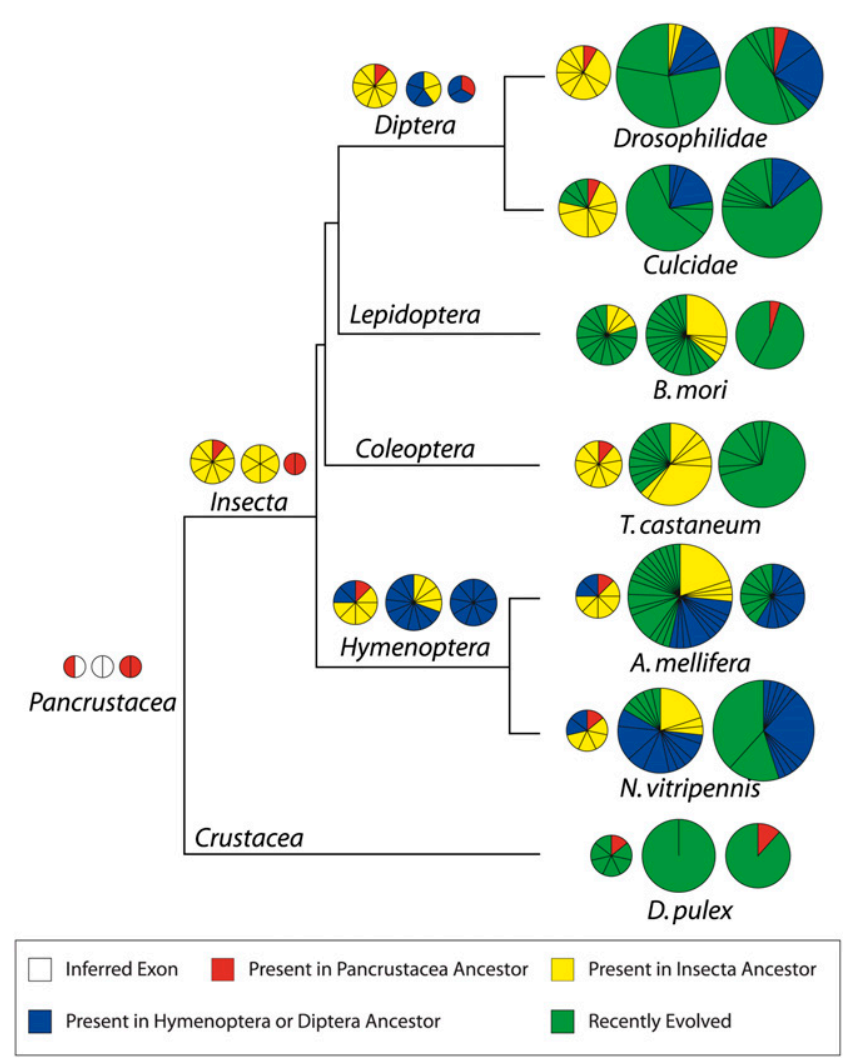

FIGURE 3. Overview of the evolution of the exon 4,6, and 9 clusters. Ancestral exons were inferred when a set of exons in a given clade (e.g., Drosophilidae) were more closely related to exon $(s)$ from a different clade (e.g., Hymenoptera) than to other exons from the same clade, based on 1000 bootstrap replicates of the phylogenetic tree (for details, see Materials and Methods). The relationship of exons in the modern species to ancestral species are depicted with pie charts (the three charts for each species or ancestors represent the exon 4, 6, and 9 clusters, respectively, from left to right). The relative number of exons in each species or ancestor is indicated by the size of the pie chart (scaled to the square root of the number of exons in the cluster). Green is used to indicate exons in the modern species that evolved since the last common ancestor and for which it is not possible to identify an ancestral exon. Blue is used to indicate exons that existed in the Diptera and Hymenoptera ancestors, but for which it is not possible to identify ancestral exons present in the Insecta ancestor. Yellow is used to indicate exons that existed in the Insecta ancestor, but for which it is not possible to identify an ancestral exon in the Pancrustacea ancestor. Red is used to indicate exons present in the Pancrustacea ancestor. White is used to represent exons that are inferred to exist in the Pancrustacea ancestor. The size of each sector of the pie graphs represents the number of exons that are ancestrally related. For example, none of the exons in the exon 9 cluster of $T$. castaneum have clear ancestral exons and therefore evolved specifically in this lineage. However, several of the T. castaneum exon 9 variants are related to one another and are therefore grouped into several sectors of the pie graph.

the Diptera ancestor. Moreover, only one duplication was detected in the exon 4 cluster between 270 and 200 mya when the Diptera diverged from the last common ancestor. Similarly, only seven duplication events were identified in the exon 4 cluster in the Lepidoptera linage during the last 270 million years. Thus, the exon 4 cluster has experienced a dramatically different evolutionary flux than the exon 6 and 9 clusters.

\section{The nature of exon duplication and loss events}

One striking feature is that nearly all of the recently duplicated exons in each cluster are adjacent in the genomic sequence. For example, three exons in the Drosophilidae exon 4 cluster (exons 4.1, 4.2, and 4.3) are recent duplications and are adjacent in the genomic sequence. This pattern holds true over all inferred duplications, with only one unambiguous exception-the duplication of exons 4.3 and 4.9 in B. mori, which are separated by five exons. It is also worth noting that all inferred duplications copied a single exon-there were no cases where two or more adjoining exons showed identical duplication patterns, suggesting that they were duplicated together. The preponderance of adjacent, single exon duplications likely reflects the molecular mechanism of duplication, which we will address later. In general, there was no evidence of other types of rearrangement; the ordering of orthologous exons was the same in all insects.

Similarly, in the exon 9 cluster, by far the predominant pattern of duplication involves single exons that are adjacent in the genomic sequence. However, a number of exceptions were observed in the exon 9 cluster. First, there are a few examples of nonadjacent pairs of duplicated exons (; e.g., D. mojavensis exons 9.19 and 9.24) (Supplemental Fig. S5). Second, there are a few cases where more than one exon was duplicated at a time. For example, in N. vitripennis exons $9.8,9.9$, and 9.10 are most similar to exons 9.14, 9.15, and 9.16, respectively (Supplemental Fig. S5B). This strongly suggests that during the evolution of

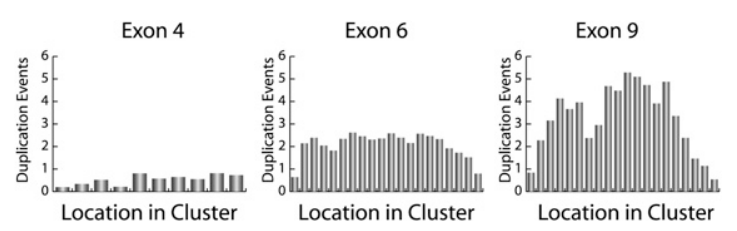

FIGURE 4. Spatial organization of exon duplications. The number of duplication events that have occurred for each exon in the exon 4, 6, and 9 clusters in D. melanogaster, A. gambiae, B. mori, T. castaneum, A. mellifera, and D. pulex during the evolution from the ancestor of all exons was calculated. For the exon 4 cluster, the exons were binned into 10 groups across the cluster (i.e., exon 4.1 was placed into bin 1 while exon 4.12 was placed into bin 10), while for the exon 6 and 9 clusters, the exons were binned into 20 groups across the cluster. Next, the average number of duplication events for all exons within each bin was calculated and graphed. The $x$-axis depicts the position of each exon in the cluster, while the $y$-axis depicts the estimated number of duplication events that have occurred on the lineage leading to this specific exon since the ancestor of all insects (for details, see the text). Noninteger values indicate uncertainty in the exact number of duplication events, based on analysis of 1000 bootstrap replicates of the phylogenetic tree for all exons in the 20 genomes for each exon cluster. 


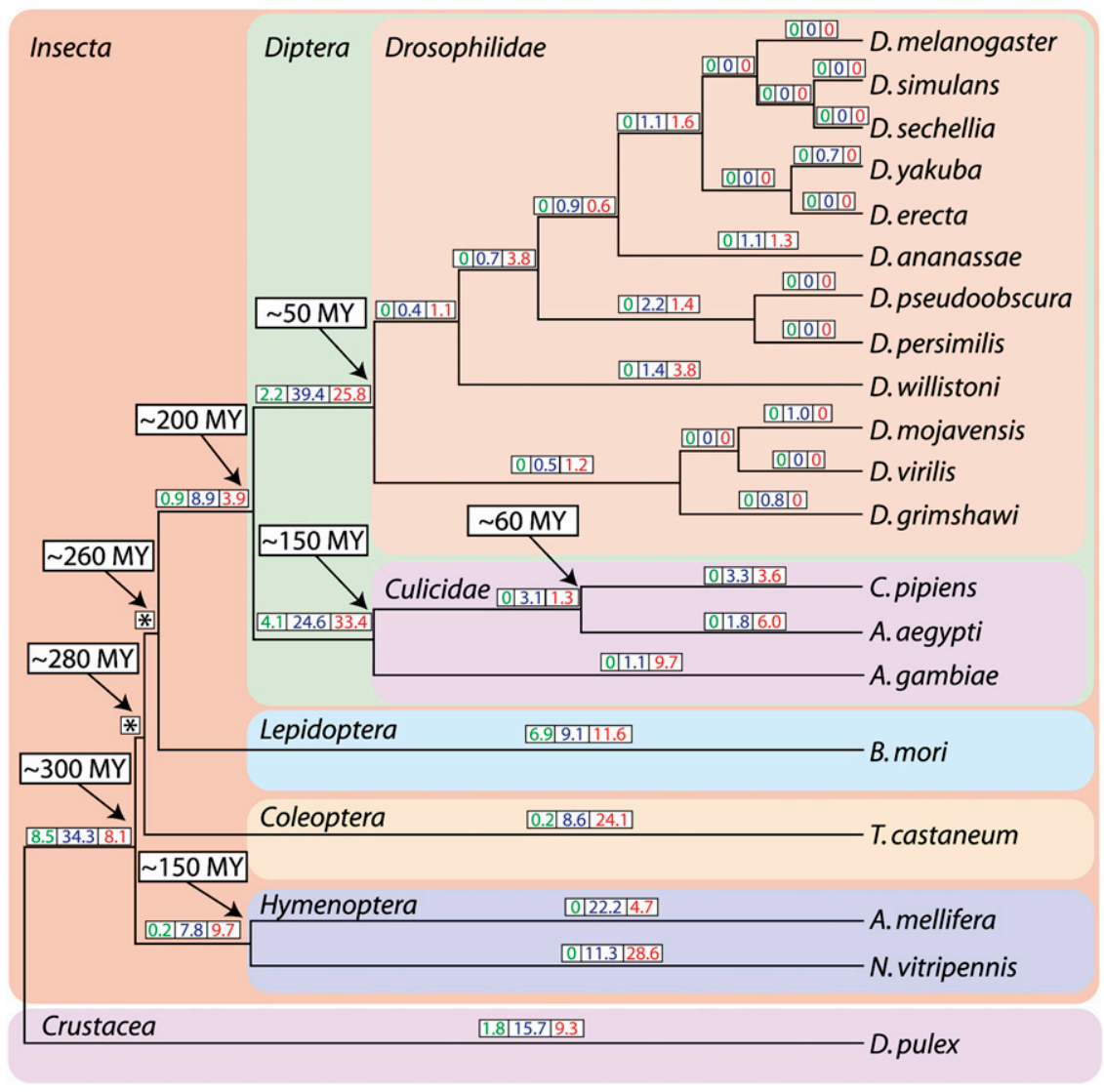

FIGURE 5. Number of exon duplication events over the course of insect evolution. The number of exon duplication events that are calculated to have occurred at each branch during evolution. The numbers of duplication events that have occurred since the last branching are depicted above each branch. The number of duplication events in the exon 4, 6, and 9 clusters are indicated in green, blue and red, respectively. The branch lengths are not drawn to indicate time (for estimated evolutionary intervals, see Fig. 1). The approximate time history at major branching events are thought to have occurred are depicted.

N. vitripennis from the Hymeopteran ancestor, three adjacent exons were simultaneously duplicated to produce exons 9.8-9.10 and 9.14-9.16. The N. vitripennis exon 9 cluster also contains an unusual region spanning from exon 9.23 to exon 9.38 in which two quite distinct groups of exons alternate in the pattern $A B A B A B A B A B A B A B$ (Supplemental Fig. S5B). It seems likely that these exons were created by several recent duplications of a pair of exons that were generated by a more ancient duplication.

Exon 6 displays the same overall patterns as exons 4 and 9. Nearly all duplications are of single exons and are adjacent, with just a few exceptions (e.g., Drosophila exons 6.3 and 6.28 [see Supplemental Fig. S4C]; N. vitripennis 6.4 and 6.26 [see Supplemental Fig. S4B]).

\section{Analysis of recent duplication and loss events}

Recent duplication events can yield insights into the molecular mechanism of duplication by providing detailed evidence of the precise boundaries of the duplicated region before mutations have had time to "scramble the evi- dence." We therefore examined in detail the most recent duplications identified in our analysis, which were within the exon 6 cluster in Drosophila species. The boundaries of these duplication events were clearly discernible. Figure 7A shows a representative example of exon duplication-D. grimshawii exon 6.47, which was recently duplicated from exon 6.46. Dynamic programming sequence alignment shows that the duplicated region extends from the $3^{\prime}$ end of exon 6.45 to the $3^{\prime}$ end of exon 6.46 . This region is $\sim 85 \%$ identical to the region from the $3^{\prime}$ end of exon 6.46 to the $3^{\prime}$ end of exon 6.47. Especially striking is the fact that not only the exons but also the corresponding intronic regions are nearly identical. Similar patterns were observed for other recent duplications such as exons 6.7/ 6.8 and 6.16/6.17 in D. ananassae, exons 6.17/6.18 in D. yakuba, and exons 6.41/ 6.42 D. pseudoobscura and D. persimilis.

Figure 7B depicts a representative example of an exon loss event. $D$. melanogaster exon 6.42 does not have an ortholog in D. yakuba, yet D. melanogaster exons 6.41 and 6.43 appear to be orthologous to D. yakuba exons 6.35 and 6.36. Moreover, the intron between D. yakuba exons 6.35 and 6.36 is highly similar to the intron between D. melanogaster exons 6.42 and 6.43. Thus, a region corresponding to D. melanogaster exon 6.42 and the upstream intron was lost in D. yakuba.

To demonstrate the statistical significance of the exon duplication results, we calculated the percentage of identity between each intron in each cassette, for all 20 species (Fig. 8 ). The distribution peaks at $\sim 40 \%$ identity and declines to near zero counts at $\sim 60 \%$ identity. By contrast the introns flanking the recently duplicated exons we identified are between $70 \%$ and $90 \%$ identical (arrows in Fig. 8). For exon 9, we identified only two cases of possibly recent exon duplication events (exons 9.17/9.21 from D. willistoni and exons 9.20/9.21 from D. pseudoobscura and D. persimilis); both were more diverged in sequence than the exon 6 duplications, and we were unable to establish unambiguous endpoints.

Several features of the recent duplication pattern are mechanistically suggestive. First, the duplicated regions correspond precisely to a unit comprised of a single intron and the downstream exon. If duplication occurred via a random mechanism, the size and boundaries of each duplication event would be different. Second, in each case the endpoints of the duplicated regions were observed to 


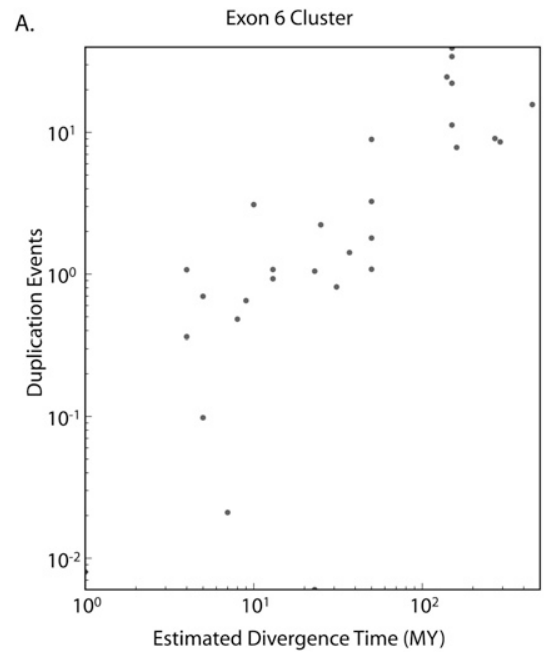

B.

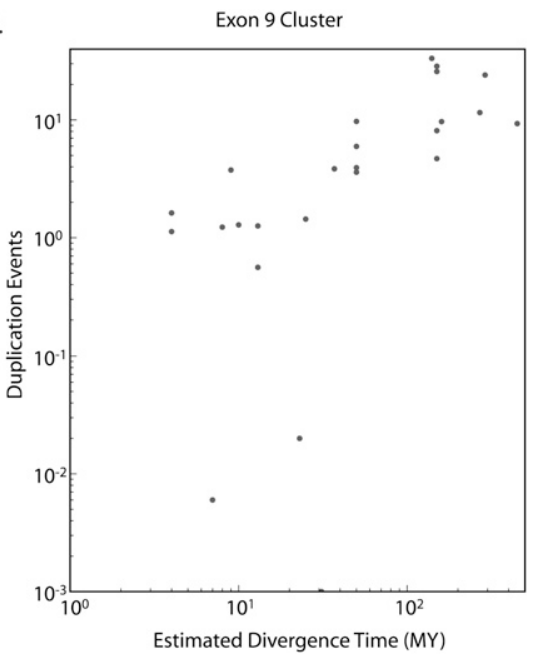

FIGURE 6. Exon duplication counts versus evolutionary branch length for the exon 6 and 9 cassettes. For each branch in the phylogeny the inferred number of exon duplications on that branch ( $y$-axis) was plotted versus the estimated length of that branch ( $x$-axis, in millions of years) based on published species phylogeny data, for cassette $6(A)$ and cassette $9(B)$. These data suggest an overall frequency of about $0.5-1$ exon duplication events per 10 million years per cassette. centers of each cluster and low levels of duplication at either end of the clusters (Fig. 4).

\section{Functional implications of the proposed duplication mechanism}

Does the proposed mechanism of staggered homologous recombination make sense in light of what is known about Dscam function, specifically its homophilic binding and mutually exclusive splicing? First, we briefly review the salient aspects of Dscam protein function. Isoform-specific homophilic binding of Dscam appears to play a crucial role in determining the patterns of neural wiring (Hughes et al. 2007; Wojtowicz et al. 2007). Dscam dimerizes with high affinity only if all three extracellular variable domains encoded be highly homologous to each other, suggesting that the molecular mechanism of duplication involves staggered homologous recombination. Pairing of homologous sequences of two adjacent exons (e.g., exons 6.45 and 6.46) would allow recombination to occur between these homologous sequences, yielding two recombination products, one containing a duplication of exon 6.46 and the preceding intron, exactly as we have observed, and the other entirely lacking exon 6.46 and the upstream intron (Fig. 9A). This hypothesis predicts that exon losses should be observed with boundaries again corresponding to a pair of homologous regions, and this is indeed what we observe. This mechanism also predicts that homologous recombination would most likely occur between adjacent exons, producing duplications that are adjacent in the genomic sequence, and this is indeed what we observed, almost without exception. Interestingly, the products of these duplication events are ideal substrates for further staggered homologous recombination events and could initiate a cascade of duplication events resulting in the serial expansion of a single exon, while other exons in the cassette either remain quiescent or are lost, consistent with what we have observed-the exon 6 and 9 clusters in each species contain large blocks of highly similar exons that are unrelated to exons in other species (Fig. 2B,C). Finally, such homologous recombination would be expected to occur most frequently at the center of the clusters or duplication blocks within each cluster, where many opportunities exist for staggered homologous pairing. By the same logic, staggered recombination would occur less frequently at the ends of each cluster, where there are fewer, if any, opportunities for staggered pairing. This corresponds precisely to what we have observed, both in terms of frequent duplications at the by the exon 4, 6, and 9 clusters match (Wojtowicz et al. 2007). For example, Dscam proteins synthesized from an mRNA containing exons 4.3, 6.10, and 9.30 ( Dscam $\left._{3.10 .30}\right)$ form homodimers but fail to bind proteins synthesized from an mRNA differing at either exon 4, 6,

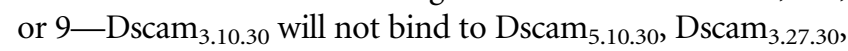
or Dscam 3.10 .25 . Crystal structures of several Dscam homodimers have revealed that the sequences within each variable

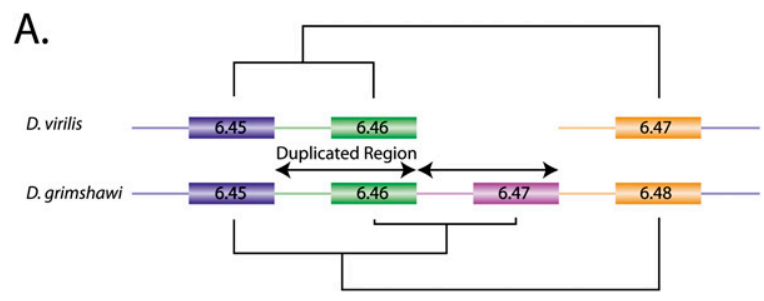

B.

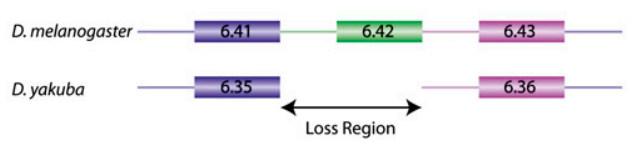

FIGURE 7. Representative recent exon duplication and loss event examples. (A) Alignment of $D$. virilis and D. grimshawii sequences shows that a new exon is present in D. grimshawii (6.47), which is not present in other Drosophila species. Alignment of the D. grimshawii sequence versus itself shows that the region from the $3^{\prime}$ end of exon 6.45 to the $3^{\prime}$ end of exon 6.46 has been duplicated, including $82 \%$ identity in the duplicated intron. (B) Alignment of D. melanogaster and $D$. yakuba sequences shows that one exon has been lost in $D$. $y a k u b a$, as indicated by a large gap extending from the $3^{\prime}$ end of exon 6.35 to the $5^{\prime}$ end of the next intron. 


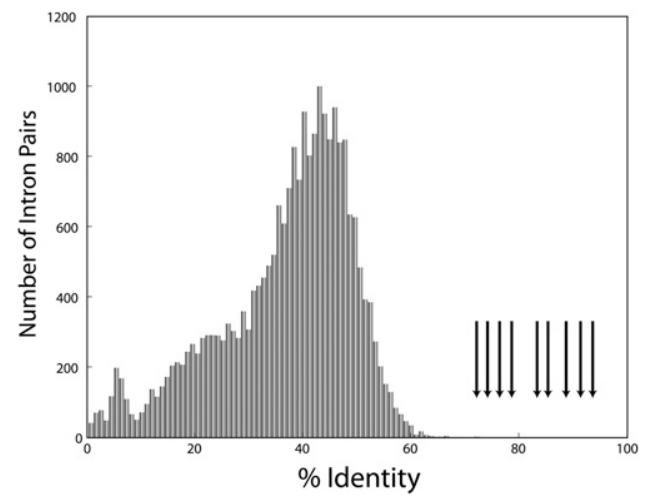

FIGURE 8. Intron-intron similarity histogram for all intron pairs in each species. The intron-intron sequence identity was calculated by global alignment of all intron-intron pairs in a given cassette in a given species, including data from all three cassettes and all 20 species. Intron pairs with very high levels of sequence identity are indicated by the arrows; e.g., D. grimshawii introns 6.45 and 6.46 display an extremely high level of identity relative to this distribution, indicating that they arose from a recent duplication (for details, see the text).

exon interact with themselves in a consistent, general, and modular way (Meijers et al. 2007; Sawaya et al. 2008).

These considerations suggest an obvious question: Would the products of the proposed staggered homologous recombination mechanism likely yield functional Dscam proteins? Specifically, would they retain homophilic binding? The most highly conserved segment of exon 6 is located at its $3^{\prime}$ end, yet all homophilic binding residues are located at the $5^{\prime}$ ends of the exons (Fig. 9B). Accordingly, staggered homologous recombination would be predicted to occur most frequently at the $3^{\prime}$ end of exon 6 and would yield duplicated exons retaining the original binding specificity (since all residues involved in binding would come from a single parent exon). Note that this matches the duplication boundaries we have observed in recent exon 6 duplications such D. grimshawii exons 6.46-6.47 (Fig. 7A). Similarly, in exon 9 the most conserved region occurs at its $3^{\prime}$ end, downstream of all the homophilic binding residues (Fig. 9B), so again duplicated exons should be functional as they would retain the original homophilic binding specificity.

Exon 4 presents a slightly more complex case, because its most conserved region lies in the center of the exon (Fig.9B, residues 12-34). Thus, the resulting recombination product would be a chimera combining parts of two separate exons (Fig. 9C), specifically the $5^{\prime}$ end of one exon, including the contact residues 1-15 (referred to as Epitope I), with the 3' half of the other exon (most likely including contact residues 27-32). However, since contact residues 27-32 are almost completely conserved over all exon 4 variants (see Fig. 9B), this chimerism would be predicted to have little effect on binding specificity. Meijers et al. (2007) have directly tested this question for one pair of exon 4 variants
(D. melanogaster exons 4.1 versus 4.9 ) by constructing chimeras consisting of residues $1-28$ of one exon joined to residues 29-68 of the other exon. Their binding data showed that the chimeras retained the binding specificity of their $5^{\prime}$ half (i.e., Epitope I). These data are consistent with our model's prediction that staggered homologous recombination products for exon 4 would usually retain homophilic binding specificity.

Another intriguing aspect of Dscam is the mechanism by which alternative splicing of the variable exon clusters occurs in a mutually exclusive manner. As mentioned earlier, the mechanism that ensures the mutually exclusive splicing exon 6 cluster involves the formation of RNA secondary structures between the docking site and a selector sequence located upstream of each exon 6 variant (Graveley 2005; Anastassiou et al. 2006). Importantly, both the docking site and the selector sequences are also present in D. sechellia, C. pipiens, N. vitripennis, and D. pulex (BR Graveley, unpubl.), species, which were not included in the initial studies that identified these elements. The second component of this system involves the RNA binding protein hrp36, which binds to the exons throughout the exon 6 cluster and represses exon inclusion (Olson et al. 2007).

Our proposed exon duplication mechanism (homologous recombination between pairs of adjacent exons) always duplicates the adjacent intron as well. Thus, all of the information required to ensure that splicing of the duplicated exon occurs in a mutually exclusive manner is simultaneously duplicated with the exon. As a result, the duplicated exon would also be spliced in a mutually exclusive manner. We imagine that there is strong selective pressure to include the selector sequence when an exon is duplicated, otherwise, splicing of the new allele would not occur in a mutually exclusive manner, resulting in the production of mRNAs encoding nonfunctional proteins.

It is well established that Dscam plays a crucial role in nervous system development (Schmucker et al. 2000; Wang et al. 2002, 2004; Hummel et al. 2003; Chen et al. 2006; Kurtz and Armitage 2006; Zipursky et al. 2006; Hattori et al. 2007; Hughes et al. 2007) and may also function in immune response in arthropods (Watson et al. 2005; Dong et al. 2006). In the immune system, it has been shown that various isoforms can physically interact with distinct pathogens (Dong et al. 2006). The sequences that are thought to be required for such interactions reside within the variable exons, but on a different surface of the protein than the homodimerization interface (Meijers et al. 2007). As a result, any newly duplicated exon would not only encode a protein capable of engaging in homotypic interactions, but would also be able to alter the pathogen recognition surface to evolve new pathogen binding specificities. It is conceivable that the rapid expansion of exons 6 and 9 in each arthropod lineage included in this study (see Fig. 5) may have been driven by selection for the ability to bind novel antigens as a part of immune system function. 


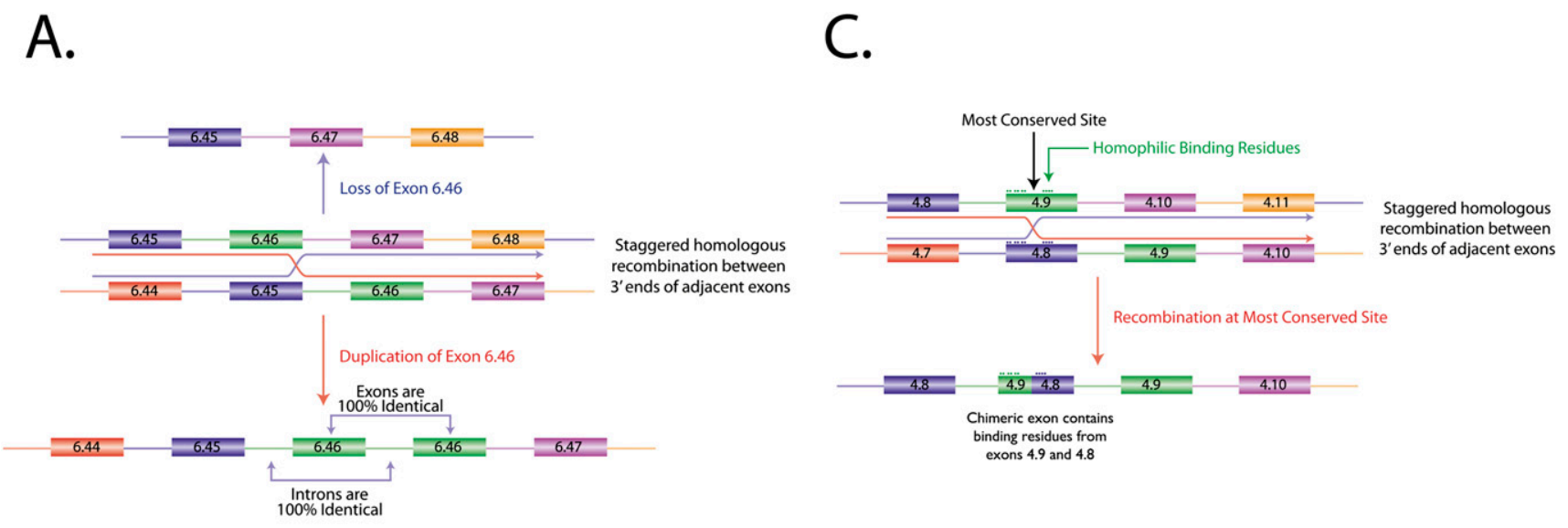

B.
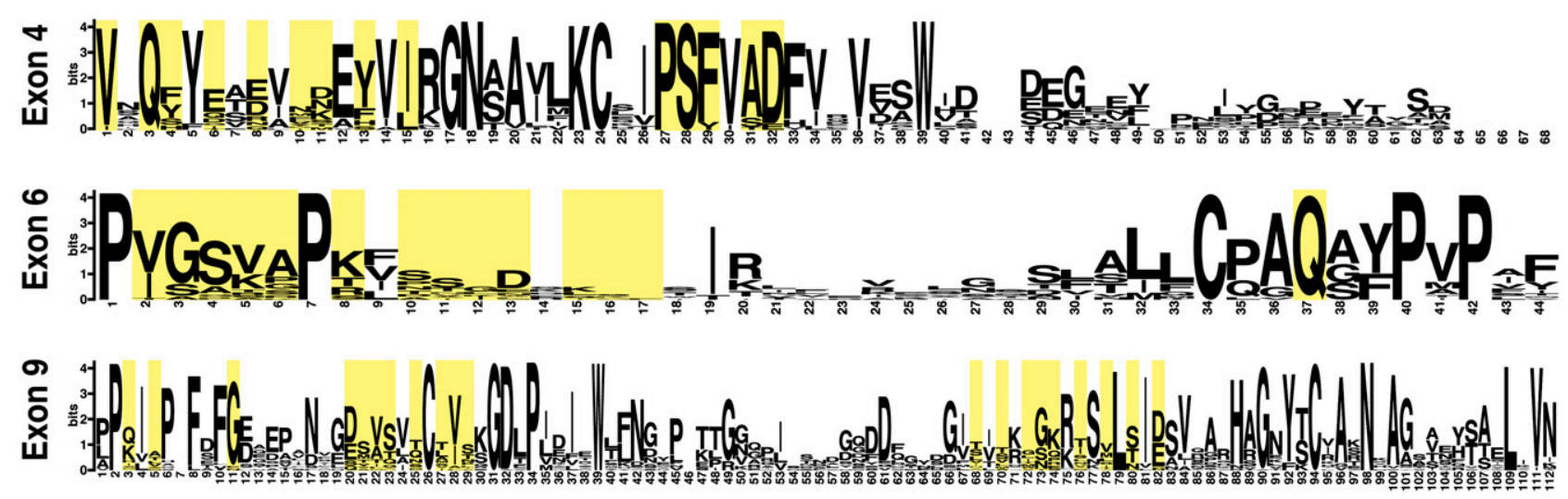

FIGURE 9. Model for the mechanism of exon creation and loss. (A) Staggered homologous recombination in the exon 6 cluster. Misalignment of similar variable exons can allow staggered homologous recombination to occur between the $3^{\prime}$ ends of adjacent exons. In this example, exons 6.44, $6.45,6.46$, and 6.47 on one chromosome misalign with exons $6.45,6.46,6.47$, and 6.48 on the other chromosome. Homologous recombination between the $3^{\prime}$ ends of exons 6.45 and 6.46 will result in the duplication of exon 6.46 on one chromosome and the deletion of exon 6.46 from the other chromosome. Importantly, when recombination occurs in this manner, both the exon and the upstream intron are duplicated. $(B)$ Pictogram representation of the protein sequences encoded by exons 4, 6, and 9 from the 20 arthropod species under study. The residues involved in homophilic binding are highlighted in yellow based on (Sawaya et al. 2008). (C) Consequences of homologous recombination in the exon 4 cluster. In this example, exons 4.7, 4.8, 4.9, and 4.10 on one chromosome misalign with exons 4.8, 4.9, 4.10, and 4.11 on the other chromosome. Homologous recombination would be most likely to occur in the middle of the exons as they are most similar. As a result, the newly created exon would be a chimera of exons 4.9 and 4.8 and would contain binding residues from both exons and may be unable to engage in homotypic binding.

\section{Concluding remarks}

We have performed a detailed analysis of Dscam throughout arthropod evolution. These studies have shown that the variable exon clusters, in particular the exon 6 and 9 clusters, have experienced numerous exon duplication and loss events since the ancestor of all insects and crustaceans. Analysis of the recent exon duplication events has revealed that in each case an intron and downstream exon were duplicated via a staggered homologous recombination event. This pattern of exon duplication simultaneously preserves the integrity of mutually exclusive splicing, the ability to engage in homotypic interactions required for Dscam function in the nervous system, and creates the potential to evolve new pathogen recognition specificities in the immune system. Thus, these studies not only provide insight into the mechanism by which Dscam rapidly evolves, but also provides a framework for understanding how complex gene structures can arise.

\section{MATERIALS AND METHODS}

\section{Data sources}

The Dscam gene sequences for A. gambiae, A. mellifera, B. mori, D. ananassae, D. erecta, D. grimshawii, D. melanogaster, D. mojavensis, D. persimilis, D. pseudoobscura, D. simulans, D. virilis, D. willistoni, D. yakuba, and T. castaneum have been described previously 
(Schmucker et al. 2000; Graveley et al. 2004; Graveley 2005; Anastassiou et al. 2006). The Dscam genes from A. aegypti, C. pipiens, D. sechelia, N. vitripennis, and D. pulex were manually assembled from individual raw sequence reads available in the NCBI trace archives in the same manner as described previously (Graveley 2005). Annotation of the genes was performed manually by a combination of cross-species and intra-species comparative genomics. Global alignment of intron sequences was performed using the EMBOSS program needle (Rice et al. 2000).

\section{Phylogeny analysis}

Nucleotide sequences for all exons were translated to amino acid sequences and aligned using CLUSTALW (Thompson et al. 1994). Sequence alignments were inspected manually and edited to correct minor gap errors (Lee and Irizarry 2001). The resulting amino acid sequence alignment was converted back to a nucleotide alignment using the RevTrans1.4 package (Wernersson and Pedersen 2003), and phylogenetic trees constructed using the standard PHYLIP programs dnadist and neighbor with default parameters (Felsenstein 1989). Bootstrap tree sampling was performed using the PHYLIP programs seqboot and consense with 1000 bootstrap replicates again with default settings. Standard measures of bootstrap confidence from these data were used throughout this study (Retief 1999) and combined with ingroup and outgroup information (i.e., the known phylogeny of the insect species) following common practice as summarized below. First, bootstrap values for monophyletic groups were determined using just the subtree of exons from a specified ingroup. Second, if outgroup exons also clustered with a given monophyletic group in more than $50 \%$ of the bootstrap replicates, they were also reported (see Tables 1-3) as evidence of a putative ancestral exon. Third, we counted duplication events using the conservative criterion of a node in the tree that connects two exons from the same species. To estimate the number of duplication events leading to a given exon (since the ancestor of all insects), we counted the number of such duplication nodes on the path from that exon up to the first ancestral node that connects it to an outgroup exon, measured over all 1000 bootstrap replicates. Similarly, we summed the count of duplication events for all exons from a given ingroup (e.g., Diptera) and subtracted the count of duplication events for its subgroups (e.g., Drosophilidae and Culicidae), to estimate the number of duplications on that branch (e.g., from the dipteran ancestor up to the divergence of Drosophilidae and Culicidae). We used a simple Python script to extract these counts from the bootstrap trees, and will make this script available upon request.

\section{Phylogeny visualization}

Data were viewed using 3D Phylogeny Explorer (Kim and Lee 2007), GeneMine (Lee and Irizarry 2001), and the Graphviz package (Gansner and North 1999).

\section{SUPPLEMENTAL MATERIAL}

Supplemental material can be found at http://www.rnajournal.org.

\section{ACKNOWLEDGMENTS}

We thank Doug Black and Larry Zipursky for discussions and comments on the manuscript. This work was supported from grants from the National Institutes of Health to C.L. (U54 RR021813) and B.R.G. (R01 GM067842). B.R.G. and C.L. conceived the work. B.R.G. assembled and annotated the Dscam genes. N.K. and C.L. contributed analysis tools. All authors analyzed the data. B.R.G. and C.L. analyzed the data, prepared the figures, and wrote the paper.

Received July 4, 2009; accepted September 15, 2009.

\section{REFERENCES}

Alekseyenko AV, Kim N, Lee CJ. 2007. Global analysis of exon creation versus loss and the role of alternative splicing in 17 vertebrate genomes. RNA 13: 661-670.

Anastassiou D, Liu H, Varadan V. 2006. Variable window binding for mutually exclusive alternative splicing. Genome Biol 7: R2. doi: 10.1186/gb-2006-7-1-r2.

Black DL. 2000. Protein diversity from alternative splicing: A challenge for bioinformatics and post-genome biology. Cell 103: 367-370.

Brett D, Hanke J, Lehmann G, Haase S, Delbruck S, Krueger S, Reich J, Bork P. 2000. EST comparison indicates 38\% of human mRNAs contain possible alternative splice forms. FEBS Lett 474: 83-86.

Brites D, McTaggart S, Morris K, Anderson J, Thomas K, Colson I, Fabbro T, Little TJ, Ebert D, Du Pasquier L. 2008. The Dscam homolog of the crustacean Daphnia is diversified by alternative splicing like in insects. Mol Biol Evol 25: 1429-1439.

Chen BE, Kondo M, Garnier A, Watson FL, Puettmann-Holgado R, Lamar DR, Schmucker D. 2006. The molecular diversity of Dscam is functionally required for neuronal wiring specificity in Drosophila. Cell 125: 607-620.

Crayton ME 3rd, Powell BC, Vision TJ, Giddings MC. 2006. Tracking the evolution of alternatively spliced exons within the Dscam family. BMC Evol Biol 6: 16.

Dong Y, Taylor HE, Dimopoulos G. 2006. AgDscam, a hypervariable immunoglobulin domain-containing receptor of the Anopheles gambiae innate immune system. PLoS Biol 4: e229. doi: 10.1371/ journal.pbio.0040229.

Felsenstein J. 1989. PHYLIP: Phylogeny inference package (version 3.2). Cladistics 5: 164-166.

Gansner ER, North SC. 1999. An open graph visualization system and its applications. Softw Pract Exper 30: 1203-1233.

Graveley BR. 2001. Alternative splicing: Increasing diversity in the proteomic world. Trends Genet 17: 100-107.

Graveley BR. 2005. Mutually exclusive splicing of the insect Dscam pre-mRNA directed by competing intronic RNA secondary structures. Cell 123: 65-73.

Graveley BR, Kaur A, Gunning D, Zipursky SL, Rowen L, Clemens JC. 2004. The organization and evolution of the dipteran and hymenopteran Down syndrome cell adhesion molecule (Dscam) genes. RNA 10: 1499-1506.

Hattori D, Demir E, Kim HW, Viragh E, Zipursky SL, Dickson BJ. 2007. Dscam diversity is essential for neuronal wiring and selfrecognition. Nature 449: 223-227.

Hughes ME, Bortnick R, Tsubouchi A, Baumer P, Kondo M, Uemura T, Schmucker D. 2007. Homophilic Dscam interactions control complex dendrite morphogenesis. Neuron 54: $417-427$.

Hummel T, Vasconcelos ML, Clemens JC, Fishilevich Y, Vosshall LB, Zipursky SL. 2003. Axonal targeting of olfactory receptor neurons in Drosophila is controlled by Dscam. Neuron 37: 221-231.

Kan Z, Rouchka EC, Gish WR, States DJ. 2001. Gene structure prediction and alternative splicing analysis using genomically aligned ESTs. Genome Res 11: 889-900. 
Kim N, Lee C. 2007. Three-dimensional phylogeny explorer: Distinguishing paralogs, lateral transfer, and violation of 'molecular clock' assumption with $3 \mathrm{D}$ visualization. BMC Bioinformatics 8: 213.

Kondrashov FA, Koonin EV. 2001. Origin of alternative splicing by tandem exon duplication. Hum Mol Genet 10: 2661-2669.

Kreahling JM, Graveley BR. 2005. The iStem, a long-range RNA secondary structure element required for efficient exon inclusion in the Drosophila Dscam pre-mRNA. Mol Cell Biol 25: 1025110260.

Kurtz J, Armitage SA. 2006. Alternative adaptive immunity in invertebrates. Trends Immunol 27: 493-496.

Lee C, Irizarry K. 2001. The GeneMine system for genome/proteome annotation and collaborative data mining. IBM Syst J 40: 592603.

Letunic I, Copley RR, Bork P. 2002. Common exon duplication in animals and its role in alternative splicing. Hum Mol Genet 11: 1561-1567.

Lev-Maor G, Sorek R, Shomron N, Ast G. 2003. The birth of an alternatively spliced exon: $3^{\prime}$ Splice-site selection in Alu exons. Science 300: 1288-1291.

Maddison WP. 1997. Gene trees in species trees. Syst Biol 46: 523-536.

Matthews BJ, Kim ME, Flanagan JJ, Hattori D, Clemens JC, Zipursky SL, Grueber WB. 2007. Dendrite self-avoidance is controlled by Dscam. Cell 129: 593-604.

Meijers R, Puettmann-Holgado R, Skiniotis G, Liu JH, Walz T, Wang JH, Schmucker D. 2007. Structural basis of Dscam isoform specificity. Nature 449: 487-491.

Mironov AA, Fickett JW, Gelfand MS. 1999. Frequent alternative splicing of human genes. Genome Res 9: 1288-1293.

Modrek B, Resch A, Grasso C, Lee C. 2001. Genome-wide analysis of alternative splicing using human expressed sequence data. Nucleic Acids Res 29: 2850-2859.

Neves G, Zucker J, Daly M, Chess A. 2004. Stochastic yet biased expression of multiple Dscam splice variants by individual cells. Nat Genet 36: 240-246.

Olson S, Blanchette M, Park J, Savva Y, Yeo GW, Yeakley JM, Rio DC, Graveley BR. 2007. A regulator of Dscam mutually exclusive splicing fidelity. Nat Struct Mol Biol 14: 1134-1140.

Pan Q, Shai O, Lee LJ, Frey J, Blencowe BJ. 2008. Deep surveying of alternative splicing complexity in the human transcriptome by high-throughput sequencing. Nat Genet 40: 1413-1415.

Park JW, Graveley BR. 2007. Complex alternative splicing. Adv Exp Med Biol 623: 50-63.

Retief JD. 1999. Phylogenetic analysis using PHYLIP. In Bioinformatics methods and protocols (ed. SAKS Misener), Vol. 132, pp. 243-258. Humana Press, New York.

Rice P, Longden I, Bleasby A. 2000. EMBOSS: The European Molecular Biology Open Software Suite. Trends Genet 16: 276-277.
Saitou N, Nei M. 1987. The neighbor-joining method: A new method for reconstructing phylogenetic trees. Mol Biol Evol 4: 406-425.

Sawaya MR, Wojtowicz WM, Andre I, Qian B, Wu W, Baker D, Eisenberg D, Zipursky SL. 2008. A double S shape provides the structural basis for the extraordinary binding specificity of Dscam isoforms. Cell 134: 1007-1018.

Schmucker D, Clemens JC, Shu H, Worby CA, Xiao J, Muda M, Dixon JE, Zipursky SL. 2000. Drosophila Dscam is an axon guidance receptor exhibiting extraordinary molecular diversity. Cell 101: 671-684.

Soba P, Zhu S, Emoto K, Younger S, Yang SJ, Yu HH, Lee T, Jan LY, Jan YN. 2007. Drosophila sensory neurons require Dscam for dendritic self-avoidance and proper dendritic field organization. Neuron 54: 403-416.

Sorek R, Lev-Maor G, Reznik M, Dagan T, Belinky F, Graur D, Ast G. 2004. Minimal conditions for exonization of intronic sequences: $5^{\prime}$ splice site formation in alu exons. Mol Cell 14: 221-231.

Thompson JD, Higgins DG, Gibson TJ. 1994. CLUSTAL W: Improving the sensitivity of progressive multiple sequence alignment through sequence weighting, position-specific gap penalties and weight matrix choice. Nucleic Acids Res 22: 4673-4680.

Wang J, Zugates CT, Liang IH, Lee CH, Lee T. 2002. Drosophila Dscam is required for divergent segregation of sister branches and suppresses ectopic bifurcation of axons. Neuron 33: 559-571.

Wang J, Ma X, Yang JS, Zheng X, Zugates CT, Lee CH, Lee T. 2004. Transmembrane/juxtamembrane domain-dependent Dscam distribution and function during mushroom body neuronal morphogenesis. Neuron 43: 663-672.

Wang ET, Sandberg R, Luo SJ, Khrebtukova I, Zhang L, Mayr C, Kingsmore SF, Schroth GP, Burge CB. 2008. Alternative isoform regulation in human tissue transcriptomes. Nature 456: 470-476.

Watson FL, Puttmann-Holgado R, Thomas F, Lamar DL, Hughes M, Kondo M, Rebel VI, Schmucker D. 2005. Extensive diversity of Igsuperfamily proteins in the immune system of insects. Science 309: 1874-1878.

Wernersson R, Pedersen AG. 2003. RevTrans: Multiple alignment of coding DNA from aligned amino acid sequences. Nucleic Acids Res 31: 3537-3539.

Wojtowicz WM, Wu W, Andre I, Qian B, Baker D, Zipursky SL. 2007. A vast repertoire of Dscam binding specificities arises from modular interactions of variable Ig domains. Cell 130: 1134-1145.

Zhan XL, Clemens JC, Neves G, Hattori D, Flanagan JJ, Hummel T, Vasconcelos ML, Chess A, Zipursky SL. 2004. Analysis of Dscam diversity in regulating axon guidance in Drosophila mushroom bodies. Neuron 43: 673-686.

Zipursky SL, Wojtowicz WM, Hattori D. 2006. Got diversity? Wiring the fly brain with Dscam. Trends Biochem Sci 31: 581-588. 

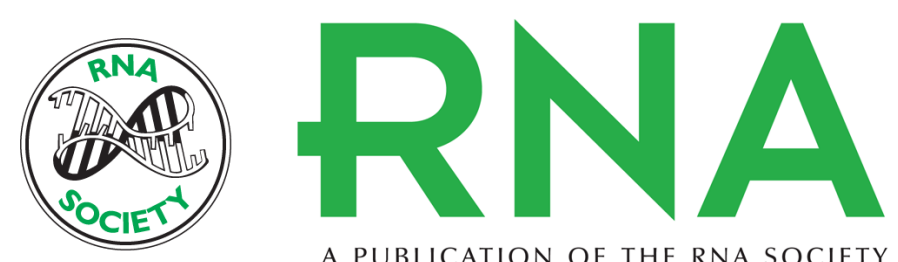

A PUBLICATION OF THE RNA SOCIETY

\section{Massive expansions of Dscam splicing diversity via staggered homologous recombination during arthropod evolution}

Christopher Lee, Namshin Kim, Meenakshi Roy, et al.

RNA 2010 16: 91-105 originally published online November 24, 2009

Access the most recent version at doi:10.1261/rna.1812710

\section{Supplemental http://rnajournal.cshlp.org/content/suppl/2009/11/10/rna.1812710.DC1 \\ Material}

References This article cites 49 articles, 7 of which can be accessed free at: http://rnajournal.cshlp.org/content/16/1/91.full.html\#ref-list-1

\section{License}

Email Alerting Receive free email alerts when new articles cite this article - sign up in the box at the Service top right corner of the article or click here.

\section{|||||||| Providing Precise Solutions for your research.}

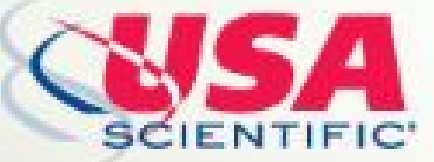

To subscribe to $R N A$ go to:

http://rnajournal.cshlp.org/subscriptions 